\title{
New Lower Bounds for Some Multicolored Ramsey Numbers
}

\author{
Aaron Robertson ${ }^{1}$ \\ Department of Mathematics, Temple University \\ Philadelphia, PA 19122 \\ email: aaron@math.temple.edu \\ Submitted: October 26, 1998; Accepted November 15, 1998 \\ Classification: 05D10, 05D05
}

\begin{abstract}
In this article we use two different methods to find new lower bounds for some multicolored Ramsey numbers. In the first part we use the finite field method used by Greenwood and Gleason [GG] to show that $R(5,5,5) \geq 242$ and $R(6,6,6) \geq 692$. In the second part we extend Fan Chung's result in [C] to show that,

$$
R\left(3,3,3, k_{1}, k_{2}, \ldots, k_{r}\right) \geq 3 R\left(3,3, k_{1}, k_{2}, \ldots, k_{r}\right)+R\left(k_{1}, k_{2}, \ldots, k_{r}\right)-3
$$

holds for any natural number $r$ and for any $k_{i} \geq 3, i=1,2, \ldots r$. This general result, along with known results, imply the following nontrivial bounds: $R(3,3,3,4) \geq 91, R(3,3,3,5) \geq 137, R(3,3,3,6) \geq 165, R(3,3,3,7) \geq 220$, $R(3,3,3,9) \geq 336$, and $R(3,3,3,11) \geq 422$.
\end{abstract}

\section{Introduction}

This paper is presented in two part which can be read independently of each other. Part one uses finite fields, and Part two extends an argument by Fan Chung.

Part one of this article is accompanied by the Maple package RES, available for download at the author's website.

Recall that $N=R\left(k_{1}, k_{2}, \ldots, k_{r}\right)$ is the minimal integer with the following property:

Ramsey Property: If we r-color the edges of the complete graph on $N$ vertices, then there exists $j, 1 \leq j \leq r$, such that a monochromatic $j$-colored complete graph on $k_{j}$ vertices is a subgraph of the r-colored $K_{N}$.

\footnotetext{
${ }^{1}$ webpage: www.math.temple.edu/ aaron

This paper is part of the author's Ph.D. thesis under the direction of Doron Zeilberger.

This paper was supported in part by the NSF under the PI-ship of Doron Zeilberger.
} 


\section{Part One: The Finite Field Method}

In this first part we add two more lower bounds to Radziszowski's Dynamic Survey $[R]$ on the subject. We show, by using the finite field technique in [GG], that $R(5,5,5) \geq 242$ and $R(6,6,6) \geq 692$. The previous best lower bound for $R(5,5,5)$ was 169 given by Song [S], who more generally shows that $R(\underbrace{5,5, \ldots, 5}_{r \text { times }}) \geq 4(6.48)^{r-1}+1$ holds for all $r$. For $R(6,6,6)$ there was no established nontrivial lower bound.

Consider the number $R(5,5,5)$. To find a lower bound, $L$, we are searching for a three coloring of $K_{L}$ which avoids a monochromatic $K_{5}$. We use an argument of Greenwood and Gleason, which is reproduced here for the sake of completeness.

Let $L$ be prime and consider the field of $L$ elements, numbered from 0 to $L-1$. Associate each field element with a vertex of $K_{L}$. We require that 3 divides $L-1$. Now consider the cubic residues of the multiplicative group $\mathbf{Z}_{L}^{*}=\mathbf{Z}_{L} \backslash\{0\}$, which form a coset of $\mathbf{Z}_{L}^{*}$. Note that since 3 divides $L-1$, there must be 2 other cosets.

Let $i$ and $j$ be two vertices of $K_{L}$. Color the edges of $K_{L}$ as follows: If $j-i$ is a cubic residue color the edge connecting $i$ and $j$ red, if it is in the second coset, color the edge blue, and if it is the third coset, color the edge green. (Note that the order of differencing is immaterial since -1 is a cubic residue.)

Now suppose that a monochromatic $K_{5}$ exists in this coloring. Without loss of generality we may call the five vertices $0, a, b, c$, and $d$, with $0<a<b<c<d$. Then the set of edges, $E=\{a, b, c, d, b-a, c-a, d-a, c-b, d-b, d-c\}$, must be a subset of one of the cosets. Since $a \neq 0$, multiplication by $a^{-1}$ is allowed. Set $B=b a^{-1}$, $C=c a^{-1}$, and $D=d a^{-1}$. Then the set $a^{-1} E=\{1, B, C, D, B-1, C-1, D-1, C-$ $B, D-B, D-C\}$ must be a subset of the cubic residues. Hence if we find an $L$ for which there does not exist $B, C$, and $D$ such that $a^{-1} E$ is a subset of the cubic residues, then we can conclude that $R(5,5,5)>L$. Of course, this argument holds for $R(\underbrace{t, t, \ldots, t}_{k \text { times }})$ for any $k$, and any $t$.

\section{Using RES}

We only acheived results when we restricted our search to fields of prime order (although any finite field can be explored using RES (or at least easily modified to do so)). Since we are considering the number $R(5,5,5)$, reject any prime, $q$, for which 3 does not divide $q-1$. This can be accomplished automaticly by using the procedure pryme. By using the procedure res we produce all of the cubic residues of $\mathbf{Z}_{p}^{*}$, for a given prime, $p$. We then use the procedure siv to discard any residue, $R$, for which $R-1$ is not a residue. We now have a much more manageable list to search. We then call the procedure diffcheck to choose all possible 3 -sets (for $B, C$, and $D$ ) and check whether or not the differences between any two elements are all cubic residues. If such a 3 -set exists, diffcheck will output the first 3-set it finds. However, in the event that no such 3 -set exists, diffcheck will output 1. 
RES can also be used to search finite fields whose order is not prime. For example, to verify that the field on $2^{4}$ elements, avoids a monochromatic triangle by using cubic residues (this fact was proven in $[G G]$ ), type GalField3 $(2,4,3)$.

By using RES we were able to find the following lower bounds: $R(5,5,5) \geq 242$ and $R(6,6,6) \geq 692$. These are obtained by the following colorings: (Since -1 is a cubic residue it suffices to list only entries up to 120 for $R(5,5,5)$ and 345 for $R(6,6,6)$.)

$R(5,5,5)>241:$

Color 1: 1, 5, 6, 8, 17, 21, 23, 25, 26, 27, 28, 30, 33, 36, 40, 41, 43, 44, 47, 48, $57,61,64,73,76,79,85,87,91,93,98,101,102,103,105,106,111,115,116,117$

Color 2: 2, 7, 9, 10, 11, 12, 16, 19, 29, 31, 34, 35, 37, 39, 42, 45, 46, 50, 52, 54, $55,56,59,60,66,67,71,72,80,82,83,86,88,89,94,95,96,113,114,119$

Color 3: 3, 4, 13, 14, 15, 18, 20, 22, 24, 32, 38, 49, 51, 53, 58, 62, 63, 65, 68, 69, $70,74,75,77,78,81,84,90,92,97,99,100,104,107,108,109,110,112,118,120$

$R(6,6,6)>691:$

Color 1: 1, 2, 4, 5, 8, 10, 16, 19, 20, 21, 25, 27, 31, 32, 33, 38, 39, 40, 42, 50, 51, $54,62,64,66,67,69,71,73,76,78,80,83,84,87,89,95,100,102,105,107,108$, $109,123,124,125,128,132,134,135,138,139,142,146,149,151,152,155,156$, $160,163,165,166,168,173,174,178,179,181,190,191,195,199,200,204,210$, $214,216,218,246,248,250,255,256,259,263,264,268,270,271,276,278,283$, 284, 291, 292, 293, 298, 301, 302, 304, 309, 310, 311, 312, 320, 326, 329, 330, 332, $333,335,336,343,345$

Color 2: 7, 9, 11, 13, 14, 17, 18, 22, 23, 26, 28, 29, 34, 35, 36, 41, 44, 45, 46, 52, $55,56,58,65,68,70,72,82,85,88,90,92,97,103,104,110,111,112,115,116,127$, $129,130,131,133,136,140,141,144,145,147,159,164,167,170,171,175,176$, $177,180,183,184,189,194,197,205,206,208,209,217,220,222,224,225,227$, $229,230,231,232,233,237,241,243,247,251,254,257,258,260,262,266,272$, $273,275,279,280,281,282,288,290,294,297,303,313,318,323,325,328,331$, $334,337,339,340,341,342$

Color 3: 3, 6, 12, 15, 24, 30, 37, 43, 47, 48, 49, 53, 57, 59, 60, 61, 63, 74, 75, 77, $79,81,86,91,93,94,96,98,99,101,106,113,114,117,118,119,120,121,122,126$, $137,143,148,150,153,154,157,158,161,162,169,172,182,185,186,187,188$, $192,193,196,198,201,202,203,207,211,212,213,215,219,221,223,226,228$, $234,235,236,238,239,240,242,244,245,249,252,253,261,265,267,269,274$, $277,285,286,287,289,295,296,299,300,305,306,307,308,314,315,316,317$, $319,321,322,324,327,338,344$ 


\section{Part Two: On the Ramsey Numbers $R\left(3,3,3, k_{1}, k_{2}, \ldots, k_{r}\right)$}

Let $N=R\left(k_{1}, k_{2}, \ldots, k_{r}\right)$. The Ramsey Property implies that there must exist a graph on $N-1$ vertices which avoids the Ramsey Property. Using such a graph, along with the construction in [C], we will prove that, for any natural number $r$ and for any $k_{i} \geq 3, i=1,2, \ldots r$,

$$
R\left(3,3,3, k_{1}, k_{2}, \ldots, k_{r}\right) \geq 3 R\left(3,3, k_{1}, k_{2}, \ldots, k_{r}\right)+R\left(k_{1}, k_{2}, \ldots, k_{r}\right)-3 .
$$

\section{The Construction}

Fix $r \geq 1$, and $k_{i} \geq 3$ for $i=1,2, \ldots r$. Let $M=R\left(3,3, k_{1}, k_{2}, \ldots k_{r}\right)$. Then there exists a graph, $G$, on $M-1$ vertices which avoids the Ramsey Property. Call the incidence matrix of this graph $T_{r+2}=T_{r+2}\left(x_{0}, x_{1}, x_{2}, \ldots, x_{r+2}\right)$, where $x_{0}$ are the diagonal entries only, and the $x_{i}$, for $i=1,2, \ldots r+2$, are the $r+2$ colors. By definition of $G$, there are no $x_{1}$-colored nor $x_{2}$-colored triangles, and no $x_{i+2}$-colored $K_{k_{i}}$, for $i=1,2, \ldots r$.

Now consider the following slightly modified construction from $[\mathrm{C}]$ :

$$
\begin{array}{ccccc}
A & & & & \\
T_{r+3}(0,1,2, \ldots, r+3)= & & & & \\
E & F & C & \\
1, \ldots, 1 & 2, \ldots, 2 & 3, \ldots, 3 & \\
\vdots & \vdots & \vdots & G \\
& 1, \ldots, 1 & 2, \ldots, 2 & 3, \ldots, 3 &
\end{array}
$$

the incidence matrix of a graph $H$ on $3 M+R\left(k_{1}, k_{2}, \ldots, k_{r}\right)-4$ vertices, where

$$
\begin{aligned}
& A=T_{r+2}(0,2,3,4,5, \ldots, r+3) \\
& B=T_{r+2}(0,3,1,4,5, \ldots, r+3) \\
& C=T_{r+2}(0,1,2,4,5, \ldots, r+3) \\
& D=T_{r+2}(3,2,1,4,5, \ldots, r+3) \\
& E=T_{r+2}(2,1,3,4,5, \ldots, r+3) \\
& F=T_{r+2}(1,3,2,4,5, \ldots, r+3)
\end{aligned}
$$

and $G$ is any matrix on $R\left(k_{1}, k_{2}, \ldots, k_{r}\right)-1$ vertices in the colors 4 through $r+3$ which avoids the Ramsey Property.

Using Fan Chung's result [C] we see that the graph $H$ avoids 1-colored, 2-colored, and 3-colored triangles. We now argue that no $(j+3)$-colored $K_{k_{j}}$ exists in $H$ for $j=$ $1,2, \ldots, r$ : Assume there exists a $J$-colored $K_{k_{J}}$ in $\mathrm{H}$, for some $J$ between 4 and $r+3$. Then there must exist $\left(\begin{array}{c}k_{J} \\ 2\end{array}\right)$ entries in the $3(M-1) \times 3(M-1)$ upper left submatrix of $T_{r+3}$, all of value $J$, which form the edges of a complete graph on $k_{J}$ vertices. However, 
by the construction of $T_{r+3}$ we see that all of these entries can be taken modulo $M-1$, since the entries of value $J$ in each block are in exactly the same places as in the upper left block, $A$. We further note that if $(s, t)$ and $(u, v)$ are two of the entries in question, then $(s, t) \not \equiv(u, v)(\bmod M-1)$ (componentwise). Without loss of generality we may assume $s<u$. If we had $s \equiv u(\bmod M-1)$, then since $(u, s)$ must also have the same value as $(u, v)$, we would have $(u, s) \equiv(u, u)(\bmod M-1)$. This implies that the entry $J$ is on the diagonal of $T_{r+2}(0,2,3,4, \ldots, r+3)$, a contradiction. Hence, if we have a $J$-colored $K_{k_{J}}$ in the upper left submatrix of $T_{r+3}$ (of size $3(M-1) \times 3(M-1)$ ), then there must be a $J$-colored $K_{k_{J}}$ in $T_{r+2}(0,2,3,4, \ldots, r+3)$, contradicting the definition of $T_{r+2}$.

Remark: Up to the renaming of colors and vertices, the above permutation configuration of colors which defines $T_{r+3}$ is the only configuration which will avoid monochromatic triangles.

\section{Harvesting Some Lower Bounds for Ramsey Numbers}

It is amazing that the result of this section has not been observed for the 25 years since $[\mathrm{C}]$ was published. Using this observation we will give 6 new lower bounds. Currently in Radziszowski's Survey $[\mathrm{R}]$, we have that $R(3,3,3,4) \geq 87$, due to Exoo [E1]. By applying the result of this section to $R(3,3,3,4)$ and using the fact that $R(3,3,4) \geq 30[\mathrm{~K}]$, we get the new lower bound: $R(3,3,3,4) \geq 91$. Using the bound $R(3,3,5) \geq 45[\mathrm{E} 2, \mathrm{KLR}]$, we get the bound $R(3,3,3,5) \geq 137$. Finally, using $R(3,3,6) \geq 54, R(3,3,7) \geq 72, R(3,3,9) \geq 110$, and $R(3,3,11) \geq 138$ all from [SLZL], we get the lower bounds $R(3,3,3,6) \geq 165, R(3,3,3,7) \geq 220, R(3,3,3,9) \geq$ 336 , and $R(3,3,3,11) \geq 422$.

\section{Acknowledgments}

I would like to thank my advisor, Doron Zeilberger, for his useful comments and insight regarding this paper. More importantly, however, I would like to thank him for sparking my interest in combinatorics and for sharing his mathematical philosophies. The mathematical community could benefit greatly from more mathematicians like him.

I would also like to thank Brendan McKay for his assistance and the referee for calling my attention to [SLZL].

\section{References}

[C] F. Chung, On the Ramsey Numbers $N(3,3, \ldots, 3 ; 2)$, Discrete Mathematics, 5, $1973,317-321$.

[E1] G. Exoo, Some New Ramsey Colorings, Electronic Journal of Combinatorics, R29, 5, 1998, 5pp.

[E2] G. Exoo, Constructing Ramsey Graphs with a Computer, Congressus Numerantium, 59, 1987, 31-36. 
[GG] R. Greenwood and A. Gleason, Combinatorial Relations and Chromatic Graphs, Canadian Journal of Mathematics, 7, 1955, 1-7.

[K] J. Kalbfleisch, Chromatic Graphs and Ramsey's Theorem, (Ph.D. Thesis), University of Waterloo, January 1966.

[KLR] D.L. Kreher, Li Wei, and S. Radziszowski, Lower Bounds for Multi-Colored Ramsey Numbers from Group Orbits, Journal of Combinatorial Mathematics and Combinatorial Computing, 4, 1988, 87-95.

[R] S. Radziszowski, Small Ramsey Numbers, Electronic Journal of Combinatorics, Dynamic Survey DS1, 1994, 28pp.

[S] Song En Min, New Lower Bound Formulas for the Ramsey Numbers $N(k, \ldots, k ; 2)$

(in Chinese), Mathematica Applicata, 6, 1993 suppl., 113-116.

[SLZL] Su Wenlong, Luo Haipeng, Zhang Zhengyou, and Li Guiqing, New Lower Bounds of Fifteen Classical Ramsey Numbers, to appear in Australasian Journal of Combinatorics. 